\title{
Perspective Trends in the Design of Multifunctional Residential Units (MRUs) in the Russian Arctic: A discussion of potentials and challenges to their implementation
}

\author{
Ilya Dunichkin ${ }^{1, *}$, Clarice Bleil de Souza ${ }^{2}$, Konstantin Bogachev ${ }^{1}$, Anna Korobeynikova ${ }^{1}$, \\ and Natalia Shchekaturova ${ }^{1}$ \\ ${ }^{1}$ Moscow State University of Civil Engineering, Yaroslavskoe shosse, 26, Moscow, 129337, Russia \\ ${ }^{2}$ Welsh School of Architecture of the Cardiff University - Bute building, King Edward VII Avenue, \\ CF10 3NB, Cardiff - UK
}

\begin{abstract}
Intensive development is carried out in the Arctic zone of the Russian Federation (AZRF) with complex modernization of transport networks and infrastructure of various types through the design, construction of Multifunctional Residential Unit (MRU) and the reconstruction of existing terminals and stations. The problem of developing new and reconstructing existing infrastructure in the Arctic has not only socio-economic, technological and planning components but fundamentally any development needs to account for extreme climatic conditions which affect urban conception and operation. This paper focuses on discussing important features of MRU developments. It shows examples of two case studies of MRU settlements considering the challenges involved in their design, further expanding the discussion in relation to the inclusion of green spaces in these settlements as well as in relation to safety and protection of pedestrians, moving between different buildings in 'enclosed' roads.
\end{abstract}

\section{Introduction}

This work discusses three important features related to the development of Arctic cities in the Russian Federation: The design and content of Multifunctional Residential Units (MRU), the importance and distribution of landscape to be used as community spaces in these settlements and issues related to pedestrian movement in extreme climate. These three features are dependent on each other and require careful consideration of design boundary conditions for indoor and space location in the urban settlement.

MRU is a modernist form of organization in the built environment. It combines various functions, from domestic to working, cultural and educational activities in a single building [1]. Multifunctionality can be seen as a necessity when cities are faced with a shortage of

\footnotetext{
Corresponding author: ecse@bk.ru
} 
territory, congestion on road and transport infrastructure and increased pace of life of urban residents as it creates a kind of "city in the city". It can be seen as a successful business model because combining several functions in a single building is cost-effective in terms of urban development.

For the settlements of the Arctic Zone of the Russian Federation (AZRF), multifunctionality is not just a 'trend' in architecture, but a vital necessity. In the conditions of the lowered Northern temperatures grouping different urban functionalities in one gives a valuable opportunity to keep people warm and to reduce time of stay outdoors. The concept of MRU is a continuation of the idea of Le Corbusier's Unite D'Habitation [2], with the difference that the main justification for their implementation in the AZRF is to protect the city as a whole, its inhabitants and any disruption on its functioning from low temperatures saving resources in building construction and operation. MRUs allow significant 'savings' in urban design through compacity of activities and clustering of infrastructure.

Multifunctional centres can also play an important role socially. At the moment, the Arctic is dominated by the phenomenon of negative demography, as well as low dynamics of natural and migration growth. Settlements have a small number of inhabitants comprising mostly people engaged in high risk activities at strategically important sites. In this situation, grouping residential cells and common recreational spaces can significantly improve the social environment. Adding green areas to recreational spaces can improve well-being, reminding people of temperate climate conditions.

Storage of vehicles and its primary maintenance is a mandatory element in a multifunctional facility. Cars need to be heated to work in the winter which can impose design constraints to car parks in terms of conditioning, infrastructure and positioning in relation to residential, office and industrial buildings. Since moving in the Arctic is also difficult, promoting accessibility to public transport either by improving existing infrastructure or developing new terminals are also part of the Arctic development agenda, meaning the connection of these facilities with MRUs needs to be considered. As most of the settlements of the Russian Arctic are working settlements, the creation of shortest possible links between functional areas can significantly improve quality of life by reducing commuting time while protected from the weather.

Therefore, designing MRUs in the arctic involves not only the grouping of basic facilities but also, the providing protected green communal spaces and safe and protected pedestrian and vehicle accessibility to different types of transport and other buildings, so the city can continue functioning in extreme winter temperatures. In the Arctic, MRU will also act as a temporary stay centre for intercity pedestrians and the connections between them need to be carefully studied to minimize pedestrian travel time between buildings, not only to promote commuting efficiency but mainly to rationalise the amount of enclosed city infrastructure for pedestrian and vehicles to reduce heating loads. However, reducing pedestrian movement time in this case involves not only choosing the shortest route but also rationalising the internal design of the MRUs and their location in the city.

This results in an iterative design process in which the internal mix of activities provided by MRUs needs to be studied together with the placement of MRUs in the city, to ensure appropriate availability of shopping, entertainment and housing, and at the same time creating centres of interaction for people rationally connected to each other. Pedestrian flow simulations are an essential component of design decision making in this case and the complexities involved in setting boundary conditions for these need to be examined considering seasonal changes and how they affect movement patterns. 


\section{Examples of MRUs in and their functionalities}

Two case studies of settlements with MRUs are critically analysed to extract lessons learned and research questions to further develop this topic as well as to propose design methods to deal with them in extreme climates.

The first case study is the Arctic Shamrock military base in the island of Alexandra Land (Fig.1). The administrative and residential complex of the base consists of four blocks on the main five-storey building and three separate "ellipses" connected to it by heated galleries with enclosed double-height spaces. The total area of the complex is $14000 \mathrm{~m} 2$. The administrative and residential complex is designed to host 150 people for 18 months and the base is self-sufficient in relation to food and fuel storage facilities. The main building of the administrative and residential complex has the shape of a shamrock - three wings connected to a central atrium. It stands on stilts buried in permafrost and is raised from the ground, allowing snow to be deposited and wind to blow underneath. The first floor is a technical floor, it hosts infrastructure and is not inhabited. In the centre of the shamrock, where all wings intersect with each other, there is an atrium, with a glazed observation deck, from which the entire territory of the base is controlled and its security is ensured [3].

\section{Premises with equipment}

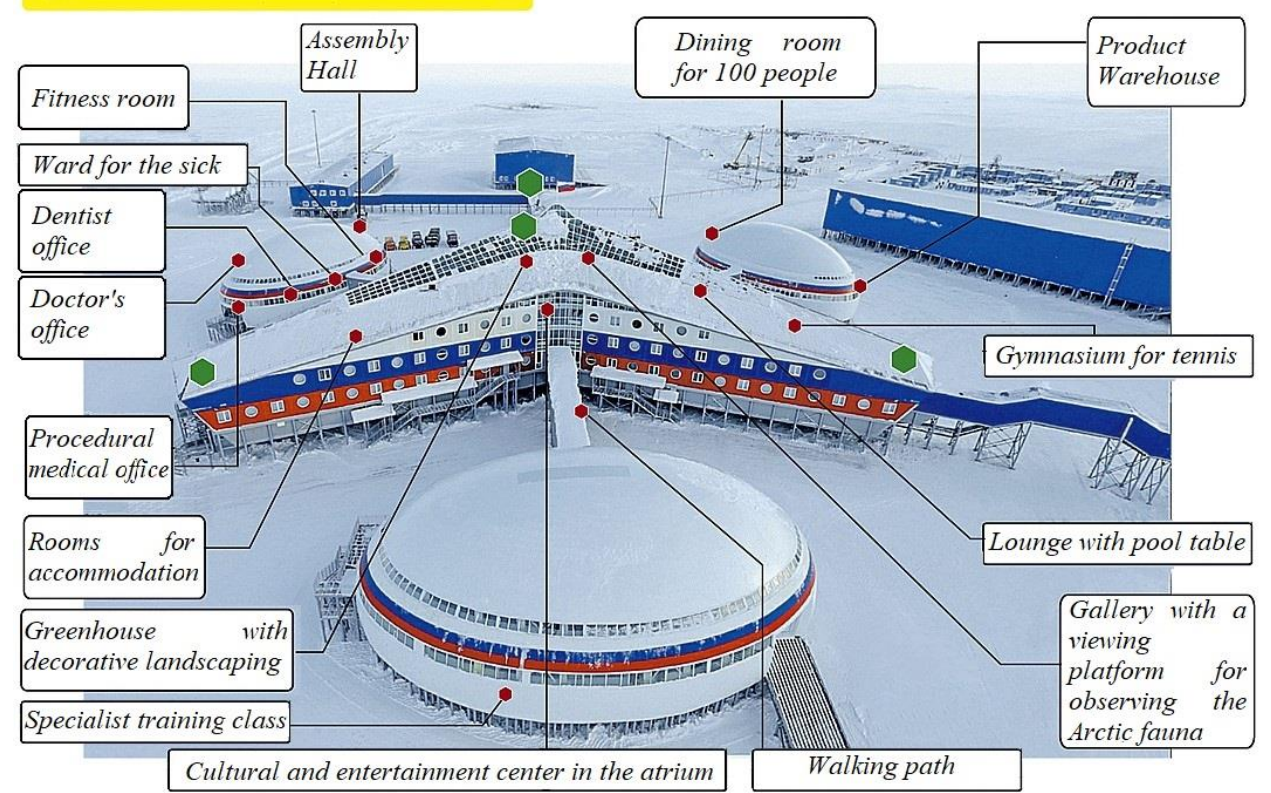

Fig. 1. General overview of the Arctic Shamrock base. Photo By Dmitry Polukhin, [3].

Despite being an interesting example of how different buildings can be connected through conditioned pedestrian routes in an efficient way, exploring the use of a simple shape, the functionality of the base is limited in relation to what it can offer in terms of lessons learned for urban development. i.e. the mix of functions is designed to attend military purposes only. This reduces the complexity of the program which therefore reduces the complexity of the layout. In reality a military base is a settlement with a clearly defined and single type of functionality, being rather different from a civilian urban settlement.

The second case study discussed is a typical project of a rotational working settlement «Tengiz» for oil production in Kazakhstan. Despite not being in the Arctic the concept can be adapted once meeting criteria to building in the permafrost. The settlement has a radial 
shape with residential areas (living cells) disposed in modular block containers connected to a central building of cylindrical shape (fig 2). The deep plan of the cylindrical building has limited connections to the outside, i.e. limited façade area, which imposes restrictions in terms of its internal layout. The mix of internal activities needs to be distributed considering limited façade availability, reducing options for community spaces which require connections with external views and daylight, such as for instance green spaces. It potentially imposes the use of a central atrium as a fixed internal layout design feature to guarantee daylight and sunlight to the inner parts of the ground floor.

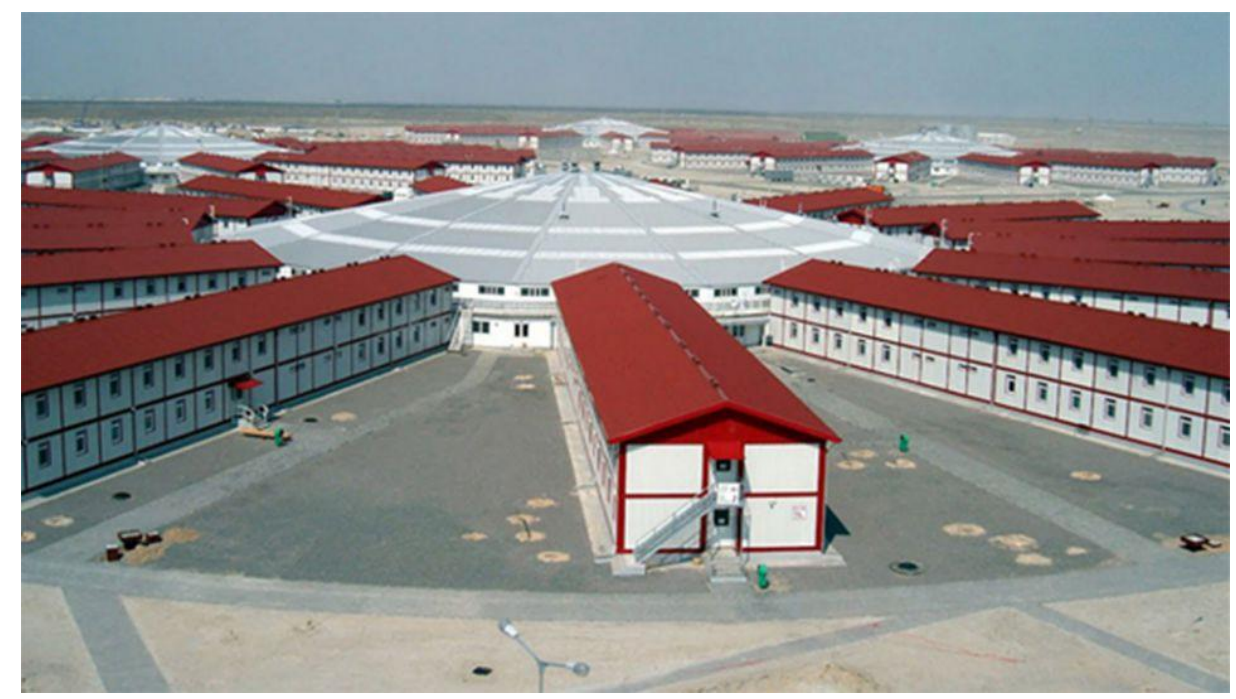

Fig. 2. Example of rotational working settlement «Tengiz» with atrium

The second example, is a less specialised type of settlement with therefore increased program complexity. Despite successfully solving the problem of connecting living spaces with communal spaces, the proposal raises questions in terms of the program and layout of communal spaces considering limitations imposed by deep plans. Limited façade availability practically imposes the use of an atrium and restricts the positioning of indoor green areas, constraining the possibility of exploring the distribution of green spaces around facades so they act as potential buffer zones and connect indoor with outdoors in the summer.

\section{Indoor gardening in MRUs}

Examples of green spaces in MRUs comprise mainly indoor roof top gardens controlled at a positive temperatures. They are normally used in waiting areas with additional services (amenities, restaurants, cafes, etc.), hotel areas and transport terminal buildings where their cost is justified and compensated by adding value to other types of profitable activities [13]. Added value to these types of activities is normally related to improving air temperature and controlling relative humidity [21] as well as bringing the feeling of temperate climate to people living in extreme conditions.

However, landscaping and greenery in MRUs can also be combined with pedestrian zones, food cord cafes, etc. [20]. They can have a potentially primary role in regulating and directing human flows not only inside MRUs but also in connections between different MRUs in a settlement. This use is currently underexplored in MRU design and could also 
be expanded if the concept of green spaces are pushed further to act as buffer zones and/or transition zones between inside and outside in summer seasons.

Depending on where they are placed, green spaces can provide interesting hybrid spaces, in which indoor conditioning in the winter can be controlled at intermediate temperatures between the comfort zone and $0^{\circ} \mathrm{C}$, whereas in the summer they enable the settlement to open to the outside and take advantage of favourable temperatures and long hours of sunshine. Hybrid spaces open the possibility for external landscapes to be explored which can make a significant contribution to improve the visual comfort of the settlements of the Russian Arctic as well as connections indoor-outdoor in favourable seasons. However, hybrid green spaces in this context, require innovative solutions to be developed and maintained using simple agricultural machinery, so maintenance can also happen when the spaces are fully enclosed.

The concept of hybrid green spaces can be pushed further to develop pedestrian routes into seasonal permeable spaces, enhancing the quality of the cities in extreme climates and at the same time reducing energy consumption for them to be run. In this case, green spaces can aid space navigation/orientation, and provide recreational areas, acting as important community hubs for interaction and wellbeing, but at the same time connecting indoors with outdoors when the climate permits. In order for this to happen, internal gardening needs to be seen as a complex, multi-stage space that needs to be considered not only in relations to its connection with the outside but also on how it affects the direction, density and speed of the human flow [22]. A rational approach to design layouts needs extensive experimentation, which calls for testing design solutions using different types of simulations. Simulations, in this case, involve assessing pedestrian flow in relation to different layout possibilities for MRU indoor mix as well as connections among different MRUs in a settlement.

\section{Methods of studying human flows in MRU}

A central feature of settlements with MRU are their interconnections. These connections need to ensure pedestrian safety against extreme climatic conditions and therefore need to be enclosed in the winter so their indoor temperature can be controlled. This mean designing MRUs requires an optimization of main pedestrian routes in terms of flow capacity and length of pathway to reduce travel time but mainly the volume of spaces to be conditioned [12]. This type of optimization calls for the use of pedestrian flow simulations in assessing design proposals.

Previously, mathematical analysis was not sufficiently used to predict and simulate the dynamics and interaction of pedestrian flows in the MRU like for transport interchange hubs [4-6]. The survey and study of public facilities showed that there is a relationship between transport and human flows, they are two mutually influencing systems [18]. The final characteristics of one of them are initial for the other, and vice versa, and set the density, speed and direction of flows from one side to the other of the settlement. For example, a massive outflow of visitors to the MRU shopping areas increase demand on pedestrian pathways to the utmost and potentially creates vehicle traffic jams the exit of parking spaces, significantly overloading road infrastructure [19]. The flow of people in this situation is a source of discomfort to the public, meaning the design of pathways needs to be based on the requirements for safety of evacuation, with flow paths sized to host maximum capacity. Insufficient considerations of overflow conditions can lead to exceeding permissible density generating human crowding or queues, reducing comfort of the pedestrian paths inside and between MRUs and creating a threat to the safety of children and people with limited mobility (PLM). As a result, both for peak and average 
flows, their permissible density should be determined taking into account the element with the lowest carrying capacity of the system.

\section{Discussion and conclusions: MRU and conditions for their development}

The analysis of MRU settlements cannot be seen disconnected from the conditions of the transport network in the Arctic. The planning structure of MRU settlements and the transport network revealed a set of conditions not yet met. The first condition is the imbalance in the pace and scale of development of different modes of transport. The most striking example is the significant lag in the development of inland water transport and the high growth rate of motorization, this affects mobility between settlements in the winter. These settlements are not prepared to receive air-cushion vehicles or different modes of transport which are more suitable to snow conditions.

The second condition is insufficient development on the existing transport infrastructure, most acutely manifested in the discrepancy between the level of road development and the level of motorization and the demand for road transport, in a sharp reduction in the number of regional and local airports, as well as in the presence of numerous "bottlenecks" at the junctions of certain modes of transport. These impose constraints in how connections between MRUs can be developed as well as on the positioning of new MRUs in the existing urban structure. The third condition is the territorial unevenness development of transport infrastructure. The far one goes on the East, the less developed are the transport infrastructure and the settlements around it. Thus, the problem of imbalance in the development of the unified transport system of Russia in the Arctic is a problem of formation of stable MRU settlements on the basis of transport hubs and adjacent residential areas.

More research is needed to balance and better match the distribution of transport interchange in the AZRF and their relationships with the promotion and development of MRU settlements so sustainable models of settlements, terminals and cities as a whole can be created. Economic sustainability could mean the promotion of trade and consumer services inside MRUs with a suitable mix of amenities to attend the demands of the MRU settlement, which do not interfere with those in transport interchange functions, promoting their role as potential regional centres. MRU have the potential to act as mini-centres within a system of settlement providing trade, consumer and leisure services. They bring daily necessary goods and services to the daily routes between home and work. This reduces regular shopping trips, decongesting streets and roads, reducing demand on public transport and in the use of cars enhancing people's safety in the harsh winters, from preventing frostbite up to enabling the continue function of basic urban facilities in seasons of heavy snow.

Environmental sustainability, on the other hand, would mean having efficient layouts which demand less energy to function in the winter. In particular, as MRUs include warm Parking, they contribute to reduce outdoor pollution as cars do not need to have their engines on during the entire cold season, when the temperature drops below $-40^{\circ} \mathrm{C}$. When green spaces are integrated to pedestrian movement and pedestrian paths and green areas are thought as buffers zones or hybrid spaces, settlements can be permeable in the summer, promoting community integration with the outdoor adding unique features to the AZRF cities.

Planning decisions related to location and interconnection of MRUs of various sizes need to be tested with the help of simulations, especially if one aims to enhance the quality of pedestrian experience and control the design and positioning of green spaces. In 
particular, the theory of human flows seems relevant to this assessment, taking into account the needs of people with limited mobility and adjustments in modelling and sizing speed of movement of people in winter clothes, a characteristic of AZRF. Since the peak pedestrian traffic can reach 35000 people, flow simulation is imperative in designing public spaces as a barrier-free environments as well as to implement techniques and special zones for the movement of people with impaired vision and other disabilities.

In a nutshell, the construction of MRU in contrast to more conventional urban approaches, especially in relation to adding mixed use activities to residential buildings, once cleverly thought in connection with transport interchange hubs and efficient hybrid green pedestrian paths, can create unique spatial features to be used in the design and urban development of cities in the AZRF, potentially providing a singular urban identity to the Northern cities of the Russian territory.

All tests were carried out using research equipment of The Head Regional Shared Research Facilities of the Moscow State University of Civil Engineering.

\section{References}

1. Desideri, U., Proietti, S., Sdringola, P., Taticchi, P., Carbone, P., \& Tonelli, F. Integrated approach to a multifunctional complex: Sustainable design, building solutions and certifications. Management of Environmental Quality: An International Journal, 21(5), 659-679. (2010)

2. Sbriglio, J., Corbusier, L., Parisis, J. L., Reyre, M., \& Gauthier, J. M. Le Corbusier: l'Unité d'habitation de Marseille. Editions Parenthèses. (1992)

3. Barenez V., In the Arctic blossomed combat "Shamrock". Newspaper "Komsomolskaya Pravda". Release from 17.04.17 (2017)

4. City-HUB, http://www.cityhub.imet.gr/, last accessed 2019/02/10

5. NODES Interchanges - New tools for designing and operation of urban transport interchanges, http://www.nodes-interchanges.eu/, last accessed 2019/02/10

6. Alliance - Enhancing excellence and innovation capacity in sustainable transport interchanges, http://alliance-project.eu/, last accessed 2019/02/10

7. R. Hickman, C.L. Chen, A. Chow, S. Saxena,: Improving interchanges in China: the experiential phenomenon. Journal of Transport Geopgraphy, 42. pp. 175-186 (2015)

8. N.G. Shishatsky, E.A. Bryukhanova, V.S. Efimov, A.M. Matveev. Strategic positioning of the Arctic region as an object of territorial development (on the example of the Khatanga-Anabar region). Arctic, (25), 150. (2016)

9. J.B. Cullingworth, Urban and regional planning in Canada. Routledge. (2017)

10. S. Hirvonen-Kantola, P. Ahokangas, M. Iivari, M. Heikkilä, H.L. Hentilä, Urban development practices as anticipatory action learning: case Arctic smart city living laboratory. Procedia Economics and Finance, 21, 337-345. (2015)

11. G.W. Johnson, A.G. Gaylord, J.C. Franco, R.P. Cody, J.J. Brady, W. Manley, C.E. Tweedie, Development of the Arctic Research Mapping Application (ARMAP): interoperability challenges and solutions. Computers \& Geosciences, 37(11), 17351742. (2011)

12. N. P. Suh: Axiomatic Design: Advances and Applications. Oxford University Press, New York (2001)

13. Jull, M. Toward a Northern Architecture: The microrayon Arctic urban prototype. Journal of Archietctural Education 70(2) 214-222. (2016)

14. N. Danilina, D. Vlasov, Aspects of transport transit hubs construction management in coordination with object lifecycle projecting. In MATEC Web of Conferences (Vol. 86, p. 05017). EDP Sciences. (2016) 
15. D. Vlasov, N. Danilina, A. Shagimuratova, The Priority Directions of Public Transport Transit Hubs Development on Commuter Railways. In Energy Management of Municipal Transportation Facilities and Transport (pp. 299-309). Springer, Cham. (2017)

16. N. Leach. Swarm urbanism. Architectural design, 79(4), 56-63. (2009)

17. A. Chan, Y. Lin. Taipei. Ant Urbanism, Neil Leach (ed.), Digital Cities, London: Wiley. (2009)

18. D. Vlasov, N. Shirokaya. Development of a polyfunctional structure of transport hubs in Smart City. In IOP Conference Series: Materials Science and Engineering (Vol. 365, No. 2, p. 022022). IOP Publishing. (2018, June)

19. V. Kholshchevnikov, D. Korolchenko, O. Zosimova, Efficiency evaluation criteria of communication paths structure in a complex of buildings of maternity and child-care institutions. In MATEC Web of Conferences (Vol. 106, p. 01037). EDP Sciences. (2017)

20. O. Korol, N. Shushunova, D. Lopatkin, A. Zanin, T. Shushunova, (2018). Application of High-tech Solutions in Ecodevelopment. In MATEC Web of Conferences (Vol. 251, p. 06002). EDP Sciences.

21. A. Krasheninnikov. Structure of social space in pedestrian realm. Architecture and modern information technologies (4), 21. (2012)

22. G.V. Esaulov. Sustainable architecture: from approaches to strategy of development. Vestnik of Tomsk State University of Architecture and Building. English version appendix, (2), 5-17. (2014)

23. V. Cherkina, N. Shushunova, J. Zubkova. Application of BIM-technologies in tasks of quality management and labour safety. In MATEC Web of Conferences (Vol. 251, p. 06004). EDP Sciences. (2018)

24. P. Kagan Monitoring of the development of urban areas with the use of information technology. In MATEC Web of Conferences (Vol. 193, p. 05031). EDP Sciences. (2018)

25. T. Vigier, D. Siret, G. Moreau, L. Lescop. Sensitive suggestion and perception of climatic effects in virtual urban environments. In Proceedings of the ACM Symposium on Applied Perception (pp. 139-139). ACM. (2013)

26. V.V. Kholshchevnikov. Experimental researches of human flow in staircases of highrise buildings. International Journal of Applied Engineering Research, 10(21), 4254942552. (2015)

27. P.C Dawson. Analysing the effects of spatial configuration on human movement and social interaction in Canadian Arctic communities. In 4th International Space Syntax Symposium (Vol. 1, pp. 37-1). (2003, June)

28. M. Shubenkov, O. Blagodeteleva. Dixon - Arctic outpost of Russia. Architecture and Modern Information Technologies, (Special Issue), 1-1. (2012) 